Article

\title{
Hierarchical Look-Ahead Conservation Voltage Reduction Framework Considering Distributed Energy Resources and Demand Reduction
}

\author{
Davye Mak (D) and Dae-Hyun Choi * \\ School of Electrical and Electronics Engineering, Chung-Ang University, 84 Heukseok-ro, Dongjak-gu, \\ Seoul 156-756, Korea; captain.davye@gmail.com \\ * Correspondence: dhchoi@cau.ac.kr; Tel.: +82-2-820-5101
}

Received: 4 October 2018; Accepted: 19 November 2018; Published: 22 November 2018

\begin{abstract}
This paper proposes a hierarchical look-ahead framework to conduct conservation voltage reduction (CVR) when distributed energy resources such as solar photovoltaic (PV) systems and energy storage systems (ESSs), and demand response programs are integrated into distribution systems. With the increasing deployment of PV systems in distribution systems, their frequently varying power output due to cloud movements could have a detrimental impact on the consumer's voltage quality, consequently leading to degraded CVR performance. A two-level CVR framework for the coordination of an on-load tap changer (OLTC), capacitor banks (CBs), and the smart inverters of PV systems/ESSs is presented, in which these elements operate to reduce the voltage profile along the distribution feeder at different temporal scales. At the global level, the operations of the OLTC and the CBs are scheduled every hour to achieve the best CVR performance in an optimization problem using mixed-integer linear programming. When voltage violations occur rapidly, the smart inverters of PV systems and ESSs help to maintain a lower voltage profile every second based on the proposed piecewise droop control functions at the local level. A simulation study is carried out in an IEEE 33-bus distribution system with an OLTC, CBs, PV systems, and ESSs, and our results demonstrate the advantages of the proposed approach in terms of voltage level and energy savings. Furthermore, the impact of demand reduction on the proposed approach is quantified, and we verify that a higher demand reduction yields more energy savings in the proposed framework.
\end{abstract}

Keywords: conservation voltage reduction (CVR); hierarchical voltage regulation; distributed energy resource (DER); demand reduction; smart inverter

\section{Introduction}

Conservation voltage reduction (CVR) is one of the main applications of Volt/VAR optimization (VVO) in distribution management systems (DMSs). CVR lowers distribution voltages to allow for consumer energy savings and peak demand reduction while keeping consumer voltages above the minimum operating limits [1]. In traditional CVR schemes, a single entity is designed to lower the voltage at all consumer feeding points in the entire distribution grid through the coordination of voltage regulators (VRs) such as on-load tap changers (OLTCs) at substations, step-type voltage regulators (SVRs), and capacitor banks (CBs), in a centralized manner. In such schemes, the centralized CVR optimization problem is formulated, where the operations of the VRs (e.g., tap changing of OLTC/SVRs and switching of CBs) are optimally scheduled for day-ahead or real-time periods.

Owing to the high penetration of distributed energy resources (DERs), such as solar photovoltaic (PV) systems, energy storage systems (ESSs), and electric vehicles (EVs) in recent years, along with various demand response (DR) programs, intermittent DER power outputs and uncertain load 
consumptions can lead to unexpected and significant voltage changes in distribution feeders. In such situations, the centralized CVR method with slow-responding VRs may not obtain the desired CVR benefits because the operation of traditional VRs is not sufficiently fast to correctly regulate voltages that quickly change because of DERs and DR. Furthermore, the computational complexity of the centralized CVR method increases as the net load profile of consumers changes frequently due to the deployment of a significant number of DERs, and due to the interaction of DRs between utilities and consumers. Our study is motivated by a desire to develop a fast and distributed CVR strategy using whose voltage along the distribution feeder can be regulated efficiently in smart distribution systems with DERs and DR.

The main objective of this paper is to propose a hierarchical multi-time scale look-ahead CVR framework for smart distribution systems. The proposed CVR framework consists of two control levels: a global level and a local level. At the global level, voltage reduction can be achieved through the coordination of a slow-responding OLTC and CBs along with the smart inverters of DERs in an optimization problem. Because rapid variations in solar power occur owing to cloud transients, only quickly responding smart inverters of DERs are controlled based on the designed droop control functions to maintain an acceptable low-voltage profile at the local level.

As an increasing number and variety of DERs and DR program participants are being connected to residential distribution grids, their flexibilities must be efficiently managed to increase the reliability of distribution grids, minimize power delivery losses from the perspective of utility, and provide high-quality electricity (in particular, high voltage quality) to consumers. The IEEE Standard 1547 for interconnection and interoperability between distribution systems and DERs was published in 2003, and the IEEE Standard 1547a, which is a revision of 1547, was announced in 2014. In IEEE Standard 1547a, DERs and DR resources are allowed to actively participate to regulate the distribution grid voltage at the point of common coupling (PCC) by changing their real and reactive power [2]. Modern DERs are equipped with advanced power electronics, namely smart inverters. They help DERs actively control their real and reactive power outputs to achieve the desired voltage regulation when abnormal voltage conditions are detected using local measurements from the DERs or smart meters in advanced metering infrastructures.

The representative method for smart inverter-based local voltage control is to use the piecewise droop control functions of DERs [3]. Droop control functions can be implemented in three modes: (1) the capacitive mode for injecting reactive power; (2) the inductive mode for absorbing reactive power; and (3) the dead-band mode, in which reactive power is neither injected nor absorbed. Based on the specified droop curves and collected local voltage measurements, the key principle of local voltage control is to quickly absorb or inject reactive power at the corresponding DERs when voltage suddenly exceeds its maximum limit or falls below its minimum limit; otherwise, no reactive power injection/absorption occurs. According to different types of measurements, such as measurements of voltage and PV power output, as well as the curtailment of real power, a variety of local voltage control methods were proposed in [4].

As the results of CVR field tests were first published in 1973 [5] along with a summary of the history of the implementation of CVR in the 1970s [6], a large body of literature on the development of CVR algorithms and their impact assessment in distribution systems has recently been accumulated. Concise summaries of implementation strategies, evaluation methods, and practical applications of CVR were presented in $[7,8]$. A review of literature related to our work can be categorized into the following three parts:

- Centralized approach: To reduce voltage in distribution systems, CVR was traditionally designed to be conducted in a centralized manner, where the CVR application in the DMS simultaneously determines the operating references for all voltage regulating devices and DERs in an optimization framework. Using mixed-integer nonlinear programming (MINLP), day-ahead models with voltage regulating devices [9] and model predictive control (MPC)-based approaches were formulated by considering solar PV and wind turbine (WT) generators [10]. From a planning 
perspective, several approaches for CVR implementation have been proposed for optimal distributed generation (DG) and ESS placement in stochastic optimization problems along with: (1) uncertain DG outputs/load consumptions [11]; (2) chance constraints [12]; and (3) the determination of the optimal location and size of capacitors and DERs in microgrids [13]. Through modeling and estimations for time-varying loads such as air conditioners and refrigerators, the impact of such loads on CVR has been quantified [14-16].

- Hierarchical distributed approach: Considerable efforts have been recently devoted to the development of distributed models and algorithms for VVO and CVR. In unbalanced three-phase distribution systems, algorithms to reduce the computational burden have been proposed based on the alternating direction method of multipliers (ADMM) [17] and a two-stage optimization problem [18]. Initially, a distributed architecture that consisted of coordinated and uncoordinated modules, corresponding to normal operation and unexpected transient cloud movements, respectively, was presented, where only traditional voltage regulation devices were used [19]. Recently, because the smart inverters of DERs can be used as fast-responding VAR supporting devices with the revised IEEE Standard 1547 for DER-grid interconnection and interoperability, a variety of multi-time scale-based distributed VVO schemes have been proposed, in which smart inverters and voltage regulating devices cooperate to smoothen the variations in voltage owing to rapidly fluctuating PV outputs and slowly changing load consumptions, respectively. These schemes include a multi-area voltage control scheme [20], a cooperative strategy between local control areas [21], a dynamic model of inverter-based DERs [22], a sensitivity analysis-based distributed control method [23], and a local power factor droop control curve [24], and the use of ESSs to improve the voltage profile [25-28]. More recently, considering uncertainties in the output of PV systems and the load demand, a robust three-stage look-ahead VVC framework was developed, in which OLTC/CBs (in the first stage) and PV inverters (in the second and third stages based on a local droop control curve) are controlled to regulate voltage [29].

- Fully distributed approach: Compared with the centralized and hierarchical distributed approaches, a fully distributed approach requires no central coordinator or distribution network model to regulate voltage. An inverter-based model-free VVO scheme has been proposed, in which each local agent is coordinated with each other to adjust their reactive power and curtail the active power in order to alleviate overvoltage phenomena [30]. In [31], a fully distributed architecture, called power electronics intelligence at the network edge (PINE), was proposed, in which the proposed PINE converters can improve the voltage profile of the end consumer without requiring a network model.

In addition to the aforementioned literature, the impact of DR on voltage quality has been studied, including the demand response mismatch (DRM) problem associated with the load's voltage dependency [32,33], a voltage unbalance mitigation strategy considering demand side management in microgrids [34], and an integrated DR and VVC algorithm in a multi-agent system [35].

However, much recent research on distributed and hierarchical VVO methods has focused only on maintaining voltage quality without explicitly considering energy savings from CVR. The primary goal of this paper is to propose a two-level CVR method applicable to smart distribution systems with high penetration of DERs and to verify the effectiveness of the proposed method using numerical examples.

The main contributions of this paper are as follows:

- We propose a distributed and hierarchical multi-time scale voltage control scheme to perform CVR in smart distribution systems. In the global control scheme, a mixed-integer linear programming (MILP)-based look-ahead optimization problem is formulated, in which the operation schedules of the voltage regulating devices (e.g., OLTC and CB) as well as PV system/ESS inverters are calculated every hour to decrease the entire feeder voltages and consequently achieve energy savings. The local control scheme involves only the inverters of the PV systems and ESSs when voltage violations occur rapidly owing to intermittent PV generation. Using the proposed CVR-based droop control curves, the amount of real and reactive powers injected into or absorbed 
by the PV systems and ESSs are determined every second to significantly mitigate quick voltage violations and achieve further CVR energy savings.

- We propose asymmetric piecewise droop control curves that can be used to conduct CVR in the local control scheme, namely a Volt-VAR curve for PV systems/ESSs and a Volt-Watt curve for the ESSs. The droop curves rely on two parameters from the global control scheme: the solution of the real/reactive power and voltage deviation from the lowest voltage limit. Based on these curves, which are updated every hour, the PV systems and ESSs perform voltage regulation every second to save energy via CVR.

Numerical examples to test and verify the performance of the proposed CVR approach are illustrated in a IEEE 33-bus distribution system with OLTC, CBs, PV systems, and ESSs. The results show that the proposed method can achieve greater energy savings than the VVO method without CVR through the optimal coordination of the OLTC, CBs, PV systems, and ESSs. We also verify that the local control scheme based on the proposed droop control functions mitigates voltage violations and further save CVR energy. Furthermore, we quantify the impact of demand reduction on the proposed method, and point out that a higher demand reduction results in greater energy savings.

The rest of this paper is organized as follows. Section 2 provides an overview of the proposed hierarchical CVR framework. Section 3 presents the formulation of the proposed CVR algorithms, which consist of a global control scheme in the MILP optimization problem and a local control scheme based on the proposed droop control functions. The simulation results for the proposed CVR approach are provided in Section 4, and the conclusions are given in Section 5.

\section{Overview of the Proposed Hierarchical CVR Framework}

The proposed hierarchical CVR framework consists of two control schemes that have multiple time scales, namely (1) a global control scheme (slow scheduling interval every hour) and (2) a local control scheme (fast scheduling interval every second), as shown in Figure 1. To achieve energy saving via voltage reduction, the global control scheme schedules the hourly operations of the OLTC, CBs, and the smart inverters of PV systems and ESSs. The global scheme is formulated in a predicted horizon-based look-ahead optimization problem with a predicted load and a PV power output, as explained in Section 3.1. In this paper, the operation times for OLTC and CB are assumed to be between $10 \mathrm{~s}$ and several minutes, which are obviously less than hourly scheduling time resolution in the global control scheme. Therefore, OLTC and CB can be successfully scheduled every hour to achieve the CVR at the global level. We also assume that the forecast error in the prediction horizon can be small by recent advanced machine learning-based forecasting technique such as deep learning method and hence do not quantify the impact of this error on the results of the proposed approach. Based on this assumption, in our simulation, load and generation data are set to average value of historical data over one-hour resolution. Load data are typical commercial energy consumption profile, and PV output data are generated according to a typical weather condition. On the other hand, the intermittent PV power output due to cloud movements could vary significantly and lead to local voltage limit violations. To resolve this issue, a local control scheme for CVR was designed and is presented in Section 3.2. In the local control scheme, by using the proposed CVR-based piecewise droop control functions, the smart inverters of the PV systems and ESSs can maintain a lower voltage profile by injecting and absorbing real/reactive power quickly. In this paper, the superscripts $*$ and $* *$ of the variables represent the optimal solutions of the global and local control schemes, respectively. 


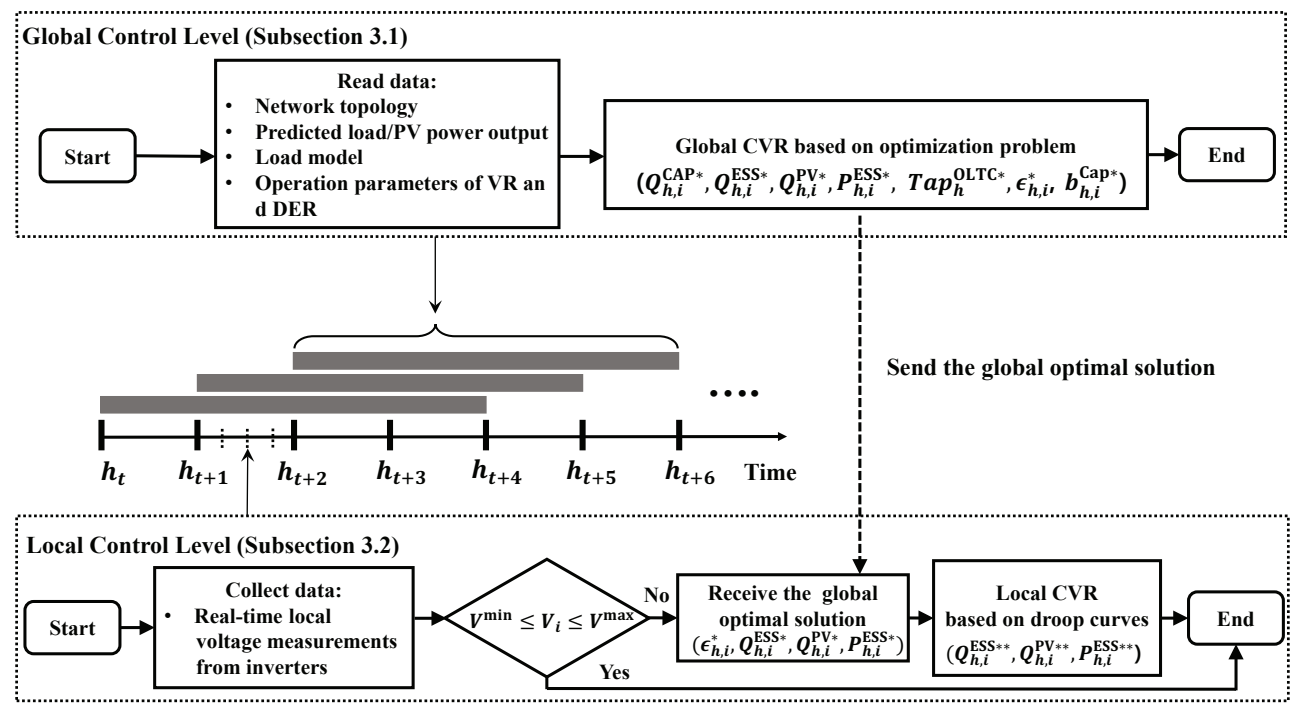

Figure 1. Flowchart of the procedure of the proposed hierarchical look-ahead conservation voltage reduction $(\mathrm{CVR})$ framework.

\section{Modeling and Solution Methodology of the Proposed CVR Framework}

\subsection{Global Control Scheme}

The global control scheme is formulated as a multi-objective optimization problem as follows:

\subsubsection{Objective Function}

For each node $i$, with scheduling period $h$ and prediction horizon $N_{h}$, the goal of the MILP-based CVR optimization problem is the minimization of the following multi objective function:

$$
\min J=\omega_{1} \sum_{h=1}^{N_{h}} \sum_{i=1}^{N_{b}} \epsilon_{h, i}+\left(1-\omega_{1}\right) \sum_{h=1}^{N_{h}} \sum_{i=1}^{N_{b}} \zeta_{h, i}
$$

In Equation (1), the first term is the total gap between the voltages and their lowest limits for all nodes during the prediction horizon, where the voltage deviation at each node has a non-negative value. The second term is the total gap between the varying state of charge (SOC) of the ESS and the middle level of the SOC. Therefore, the optimization problem focuses on minimizing these two objective functions simultaneously to obtain the desired CVR benefits through voltage reduction while preventing the SOC from reaching its upper or lower limits quickly. The positive parameters $\omega_{1}\left(0 \leq \omega_{1} \leq 1\right)$ and $1-\omega_{1}$ are penalty terms for voltage reduction and SOC regulation, respectively. A larger $\omega_{1}$ leads to a larger $\epsilon_{h, i}$ along with greater voltage reduction, thus resulting in increasing the consumer's energy savings, but limiting the charge and discharge of the ESS for voltage regulation because of the smaller penalty parameter $1-\omega_{1}$. Using the previously described objective function, the proposed CVR optimization problem can be formulated with the following linear equality/inequality constraints.

\subsubsection{CVR Constraints}

According to the American National Standards Institute (ANSI) standard C84.1, voltages for residential customers should be maintained at $120 \mathrm{~V} \pm 5 \%(114-126 \mathrm{~V})$. The key principle of CVR is to limit the voltage profile to within the lower half range (114-120 V) to reduce energy consumption and achieve the desired quality of voltage. In this context, the voltage constraint considering CVR operation is defined as

$$
\epsilon_{h, i}=V_{h, i}-V_{\min }, \epsilon_{h, i} \geq 0 .
$$


In the objective function Equation (1), the minimization of $\epsilon_{h, i}$ guarantees that the voltage profile at any node $i$ will become as low as possible according to its minimum limit, thus maximizing the effects of CVR.

\subsubsection{Distribution Power Flow and Load Model}

Equations (3), (4), and (5) represent the linearized distribution real power flow, reactive power flow, and voltage for node $i$ at period $h$, respectively [36]:

$$
\begin{aligned}
& P_{h, i+1}^{\text {line }}=P_{h, i}^{\text {line }}-P_{h, i+1}^{\text {node }}-P_{h, i+1}^{\text {lat }} \\
& Q_{h, i+1}^{\text {line }}=Q_{h, i}^{\text {line }}-Q_{h, i+1}^{\text {node }}-Q_{h, i+1}^{\text {lat }} \\
& V_{h, i+1}=V_{h, i}-\left(\frac{r_{i} P_{h, i}^{\text {line }}+x_{i} Q_{h, i}^{\text {line }}}{V_{h, 1}}\right) .
\end{aligned}
$$

Equation (6) represents substation voltage, which can be determined by the OLTC tap position $\mathrm{Tap}_{h}^{\mathrm{OLTC}}$, along with the step size used to change tap positions $a^{\text {OLTC: }}$

$$
V_{h, 1}=V^{\text {nom }}+a^{\text {OLTC }} \operatorname{Tap}_{h}^{\text {OLTC }} .
$$

For each node $i$, the nodal real and reactive power balance equations can be expressed in terms of the real and/or reactive power of the load, CBs, and DERs as follows:

$$
\begin{aligned}
& P_{h, i}^{\text {node }}=P_{h, i}^{\text {load }}-\widehat{P}_{h, i}^{\mathrm{PV}}-P_{h, i}^{\mathrm{ESS}, \mathrm{dch}}+P_{h, i}^{\mathrm{ESS}, \mathrm{ch}}, \\
& Q_{h, i}^{\text {node }}=Q_{h, i}^{\text {load }}-Q_{h, i}^{\mathrm{PV}}-Q_{h, i}^{\mathrm{ESS}}-Q_{h, i}^{\mathrm{CAP}} .
\end{aligned}
$$

In Equations (7) and (8), the real and reactive powers of load consumption are defined based on an exponential load model

$$
\begin{aligned}
P_{h, i}^{\text {load }} & =P_{h, i}^{\text {load,nom }}\left(1-X_{h, i}^{\mathrm{DR}}\right)\left(V_{h, i}\right)^{\alpha}, \\
Q_{h, i}^{\text {load }} & =Q_{h, i}^{\text {load,nom }}\left(1-X_{h, i}^{\mathrm{DR}}\right)\left(V_{h, i}\right)^{\beta} .
\end{aligned}
$$

Here, each of the real and reactive load consumptions depends on its corresponding voltage and certain specified exponents, $\alpha$ and $\beta$, respectively. In Equations (9) and (10), $X_{h, i}^{\mathrm{DR}}$ indicates the amount of load reduction within [0,1], obtained via the DR program. In the scope of our work, load reduction can be carried out by controllable appliances such as air conditioners. For example, if $X_{h, i}^{\mathrm{DR}}=0$, no load reduction occurs for node $i$ at period $h$. Some controllable appliances should turn off when $X_{h, i}^{\mathrm{DR}}$ is greater than zero.

To formulate the proposed CVR algorithm in the MILP optimization problem, the nonlinear equations for load consumption Equations (9) and (10) have to be linearized. To this end, the piecewise linear approximation method is adopted as follows:

$$
\begin{aligned}
& P_{h, i}^{\text {load }}=P_{h, i}^{\text {load,nom }}\left(1-X_{h, i}^{\mathrm{DR}}\right)\left(\sum_{k \in K_{i}} S_{h, i, k}^{P} \Delta V_{h, i}^{k}\right), \\
& Q_{h, i}^{\text {load }}=Q_{h, i}^{\text {load,nom }}\left(1-X_{h, i}^{\mathrm{DR}}\right)\left(\sum_{k \in K_{i}} S_{h, i, k}^{Q} \Delta V_{h, i}^{k}\right),
\end{aligned}
$$




$$
\begin{aligned}
& 0 \leq \Delta V_{h, i}^{k} \leq \frac{V_{h, i}^{k, \max }}{K}, \\
& V_{h, i}=\sum_{k=1}^{K} \Delta V_{h, i}^{k} \\
& \Delta V_{h, i}^{k} \leq \Delta V_{h, i}^{k-1}, \quad k=2, \ldots, K, \\
& \frac{V_{h, i}^{k, \max }}{K}-\Delta V_{h, i}^{k-1} \leq b_{h, i}^{k-1, p} \frac{V_{h, i}^{k, \max }}{K}, k=2, \ldots, K, \\
& \Delta V_{h, i}^{k} \leq\left(1-b_{h, i}^{k-1, p}\right) \frac{V_{h, i}^{k, \max }}{K}, \quad k=2, \ldots, K,
\end{aligned}
$$

where

$$
\begin{aligned}
& S_{h, i, k}^{P}=\frac{\left(V_{h, i}^{k}\right)^{\alpha}-\left(V_{h, i}^{k-1}\right)^{\alpha}}{V_{h, i}^{k}-V_{h, i}^{k-1}}, \\
& S_{h, i, k}^{Q}=\frac{\left(V_{h, i}^{k}\right)^{\beta}-\left(V_{h, i}^{k-1}\right)^{\beta}}{V_{h, i}^{k}-V_{h, i}^{k-1}} .
\end{aligned}
$$

In Equations (9) and (10), the exponential voltage terms for the real and reactive load consumption can be approximated via piecewise linearization, expressed in Equations (11) and (12). For a total of $K$ breaking points, $\Delta V_{h, i}^{k}$ is the increment in $V_{h, i}$ in the $k$ th piecewise interval $(k=1, \ldots, K)$ in Equation (13), and the sum of $\Delta V_{h, i, k}$ for all piecewise blocks is defined as $V_{h, i}$ in Equation (14). Equations (15), (16), and (17) ensure that each piecewise linear block is sequentially filled up with $\Delta V_{h, i, k}$ from left to right. The slopes of the $k$ th piecewise linear block for real and reactive load consumption are described in Equations (18) and (19).

\subsubsection{ESS Operation}

For the ESS at node $i$, Equation (20) defines the operational dynamics of its SOC at the current hour $h$ in terms of its SOC at the previous hour $h-1$, its battery capacity $E_{i}^{\mathrm{ESS}, \max }$, its charging and discharging efficiency, $\eta_{i}^{\mathrm{ch}}$ and $\eta_{i}^{\mathrm{dch}}$, and its charging and discharging power, $P_{h, i}^{\mathrm{ESS}, \mathrm{ch}}$ and $P_{h, i}^{\mathrm{ESS}, \mathrm{dch}}$. The capacity constraint of the SOC is presented in Equation (21):

$$
\begin{gathered}
\mathrm{SOC}_{h, i}=S O C_{h-1, i}+\frac{\eta_{i}^{\mathrm{ch}} P_{h, i}^{\mathrm{ESS}, \mathrm{ch}}}{E_{i}^{\mathrm{ESS}, \mathrm{max}}}-\frac{P_{h, i}^{\mathrm{ESS}, \mathrm{dch}}}{\eta_{i}^{\mathrm{dch}} E_{i}^{\mathrm{ESS}, \max }}, \\
S O C_{h, i}^{\min } \leq S O C_{h, i} \leq S O C_{h, i}^{\max }, \\
P_{i}^{\mathrm{ESS}, \mathrm{ch}, \mathrm{min}} b_{h, i}^{\mathrm{ESS}} \leq P_{h, i}^{\mathrm{ESS}, \mathrm{ch}} \leq P_{i}^{\mathrm{ESS}, \mathrm{ch}, \max _{h, i}^{\mathrm{ESS}},} \\
P_{i}^{\mathrm{ESS}, \mathrm{dch}, \min }\left(1-b_{h, i}^{\mathrm{ESS}}\right) \leq P_{h, i}^{\mathrm{ESS}, \mathrm{dch}} \leq P_{i}^{\mathrm{ESS}, \mathrm{dch}, \max }\left(1-b_{h, i}^{\mathrm{ESS}}\right) .
\end{gathered}
$$

It should be noted that, in Equation (21), the SOC is bounded by

$$
\begin{aligned}
S O C_{h, i}^{\min } & =\max \left\{S O C_{i}^{\mathrm{mid}}-\zeta_{h, i}, S O C_{i}^{\min }\right\}, \\
S O C_{h, i}^{\max } & =\min \left\{S O C_{i}^{\mathrm{mid}}+\zeta_{h, i} S O C_{i}^{\max }\right\}, \\
\zeta_{h, i} & \geq \zeta^{\text {th }},
\end{aligned}
$$

where the SOC regulation variable $\zeta_{h, i}$ determines the range of the SOC with a predefined threshold $\zeta^{\text {th }}$ for node $i$ at every hour $h$. Equations (22) and (23) are constraints on the charging and discharging powers of the ESS, where $b_{h, i}^{\mathrm{ESS}}$ represents a binary decision variable that determines the on/off status 
of the ESS. No reserved charging real power is required in Equation (22). In contrast, the maximum discharging real power is limited in Equation (23) by:

$$
P_{h, i}^{* \mathrm{ESS}, \mathrm{dch}, \max }=P_{i}^{\mathrm{ESS}, \mathrm{dch}, \max }-P_{i}^{\mathrm{ESS}, \mathrm{res},}
$$

where $P_{i}^{\mathrm{ESS} \text {,res }}$ is the reserved real power for the local control scheme that is described in the following subsection.

\subsubsection{Reactive Power Capability of DERs}

The reactive power capability of the PV systems and the ESS at node $i$ can be described in terms of the minimum power factor $\left(P F^{\mathrm{PV}, \mathrm{min}}, P F^{\mathrm{ESS}, \mathrm{min}}\right)$, the predicted $\mathrm{PV}$ real power output $\left(\widehat{P}_{h, i}^{\mathrm{PV}}\right)$, the charging/discharging power of the ESS $\left(P_{h, i}^{\mathrm{ESS}, \mathrm{ch}}, P_{h, i}^{\mathrm{ESS}, \mathrm{dch}}\right)$, and the reserved reactive power $\left(Q_{i}^{\mathrm{PV}, \text { res }}\right.$, $\left.Q_{i}^{\mathrm{ESS}, \text { res }}\right)$. In particular, the solution for the reactive power of DER at the global control level is embedded into the local droop curves.

$$
\begin{aligned}
-\left(c^{\mathrm{PV}} \hat{P}_{h, i}^{\mathrm{PV}}-Q_{i}^{\mathrm{PV}, \text { res }}\right) & \leq Q_{h, i}^{\mathrm{PV}} \leq c^{\mathrm{PV}} \widehat{P}_{h, i}^{\mathrm{PV}}-Q_{i}^{\mathrm{PV}, \text { res }} \\
-\left({ }^{\mathrm{ESS}} P_{h, i}^{\mathrm{ESS}, \mathrm{dch}}-Q_{i}^{\mathrm{ESS}, \mathrm{res}}\right) & \leq Q_{h, i}^{\mathrm{ESS}} \leq c^{\mathrm{ESS}} P_{h, i}^{\mathrm{ESS}, \mathrm{dch}}-Q_{i}^{\mathrm{ESS}, \text { res }} \\
-\left({ }^{\mathrm{ESS}} P_{h, i}^{\mathrm{ESS}, \mathrm{ch}}-Q_{i}^{\mathrm{ESS}, \text { res }}\right) & \leq Q_{h, i}^{\mathrm{ESS}} \leq c^{\mathrm{ESS}} P_{h, i}^{\mathrm{ESS}, \mathrm{ch}}-Q_{i}^{\mathrm{ESS}, \text { res }},
\end{aligned}
$$

where

$$
\begin{aligned}
c^{\mathrm{PV}} & =\sqrt{\frac{1-\left(P F^{\mathrm{PV}, \mathrm{min}}\right)^{2}}{\left(P F^{\mathrm{PV}, \mathrm{min}}\right)^{2}}}, \\
c^{\mathrm{ESS}} & =\sqrt{\frac{1-\left(P F^{\mathrm{ESS}, \mathrm{min}}\right)^{2}}{\left(P F^{\mathrm{ESS}, \mathrm{min})^{2}}\right.} .}
\end{aligned}
$$

In this study, the power factor at any interconnected point between the grid and the DERs is assumed to be between 0.9 leading and 0.9 lagging.

It should be noted that some amount of the available real and reactive power of the DERs needs to be reserved at the global level for local control. If no power is reserved for the PV systems and ESSs, they may not be able to resolve rapid violations of local voltages owing to the cloud transients because all of their voltage regulating capabilities will be involved at the global control level. The reserved power for the local control can be calculated using the following equations:

$$
\begin{aligned}
Q_{h, i}^{\mathrm{res}} & =\frac{1}{N_{b}-1} \frac{\partial Q_{h, i}}{\partial V_{h, i}}\left(V^{\mathrm{min}}-V_{i}^{\mathrm{th}}\right), \\
P_{h, i}^{\mathrm{res}} & =\frac{1}{N_{b}-1} \frac{\partial P_{h, i}}{\partial V_{h, i}}\left(V^{\mathrm{min}}-V_{i}^{\mathrm{th}}\right),
\end{aligned}
$$

where $V_{i}^{\text {th }}$ in the local droop curve is set to determine the reserved power for each DER. As the value of $V_{i}^{\text {th }}$ decreases (or increases), more (or less) reserved power is required. More reserved power allows for greater flexibility in the local CVR control. However, it also allows for more limited voltage regulating capabilities for the DER in the global control level, consequently degrading the performance of the global optimization. The value of $V_{i}^{\text {th }}$ can be determined by system operators according to weather and loading conditions. In addition, $\epsilon_{h, i}^{*}$, which is calculated at the global level, is also embedded into the local droop curves, with which the voltage is regulated to keep it below the value of $V^{\min }+\epsilon_{h, i}^{*}$. 


\subsubsection{Voltage Regulating Device Operation}

For the CB at node $i$, its reactive output is calculated at every scheduling period $h$ as follows:

$$
Q_{h, i}^{\mathrm{CAP}}=b_{h, i}^{\mathrm{Cap}} Q_{i}^{\mathrm{CAP}, \mathrm{nom}},
$$

where $Q_{i}^{\text {CAP,nom }}$ is the size of the capacitors and $b_{h, i}^{\text {Cap }}$ is a binary decision variable that determines the switch status of the capacitors. During the prediction horizon $N_{h}$, the total number of switching operations for the OLTC and CBs is limited by their corresponding switching thresholds, NTa $p_{\max }^{\text {OLTC }}$ and NCap $\max$ :

$$
\begin{array}{r}
\sum_{h=1}^{N_{h}} \mid \text { Tap }_{h}^{\text {OLTC }}-\text { Tap }_{h-1}^{\text {OLTC }} \mid \leq \text { NTap }_{\max }^{\text {OLTC }} \\
\sum_{h=1}^{N_{h}}\left|b_{h, i}^{\text {Cap }}-b_{h-1, i}^{\text {CAP }}\right| \leq \text { NCap }_{\max .}
\end{array}
$$

In the formulation of the MILP optimization problem, the nonlinear constraints expressed in Equations (36) and (37) can be linearized as

$$
\begin{aligned}
& N \operatorname{Nap}_{h}^{\mathrm{OLTC}} \geq \operatorname{Tap}_{h}^{\mathrm{OLTC}}-\operatorname{Tap}_{h-1}^{\mathrm{OLTC}}, \\
& \operatorname{NTap}_{h}^{\mathrm{OLTC}} \geq \operatorname{Tap}_{h-1}^{\mathrm{OLTC}}-\operatorname{Tap}_{h}^{\mathrm{OLTC}}, \\
& \sum_{h=1}^{N_{h}} \text { NTap }_{h}^{\text {OLTC }} \leq \text { NTap }_{\max }^{\text {OLTC }} \\
& \operatorname{NCap}_{h, i} \geq b_{h, i}^{\text {Cap }}-b_{h-1, i^{\prime}}^{\text {Cap }} \\
& \operatorname{NCap}_{h, i} \geq b_{h, i}^{\mathrm{Cap}}-b_{h-1, i^{\prime}}^{\mathrm{Cap}} \\
& \sum_{h=1}^{N_{h}} N \operatorname{Cap}_{h, i} \leq \text { NCap }_{\max }
\end{aligned}
$$

where the linear constraints in Equations (38), (39) and (40) and (41), (42) and (43) correspond to the nonlinear constraints shown in Equations (36) and (37), respectively.

Finally, the range of allowable voltages for all nodes can be expressed as

$$
V^{\min } \leq V_{h, i} \leq V^{\max }
$$

where $V^{\min }$ and $V^{\max }$ are selected to be 0.95 p.u. and 1.05 p.u.

\subsection{Local Control Scheme}

In this subsection, the local voltage control scheme is described, in which the real and reactive power outputs are locally injected or absorbed via the operation of the smart inverters of the PV systems and ESSs based on the proposed droop control functions. It should be noted that sudden changes in weather conditions (e.g., intermittent cloud movements) generate uncertain fluctuations in the output of the PV system, leading to fast and unexpected voltage violations at the points of interconnection between the grid and the PV systems. In such a scenario with transient clouds, the fast-responding smart inverters of DERs are more effective at rapidly mitigating voltage violations than the slow-responding VRs.

The proposed local control scheme is initiated when a voltage violation is detected via real-time local voltage measurements at the smart inverter. The smart inverter then starts providing further power support quickly according to the collected local voltage measurements and its control settings. 
To achieve the desired CVR operation, two droop curves, corresponding to Volt-VAR control for PV systems and ESSs and Volt-Watt control for ESSs, are proposed as shown in Figure 2a,b.

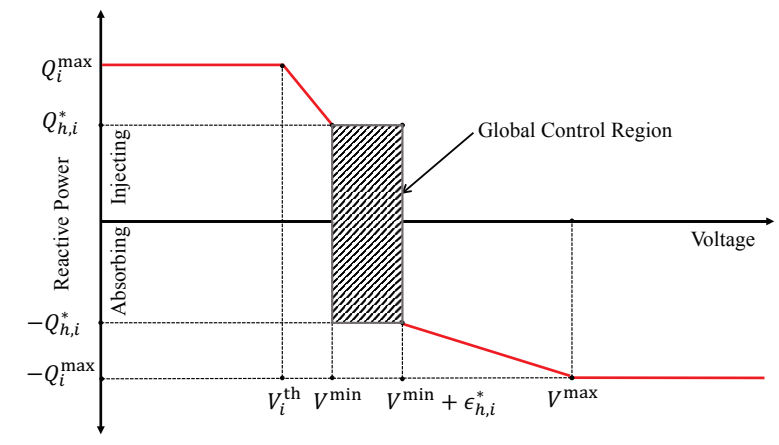

(a)

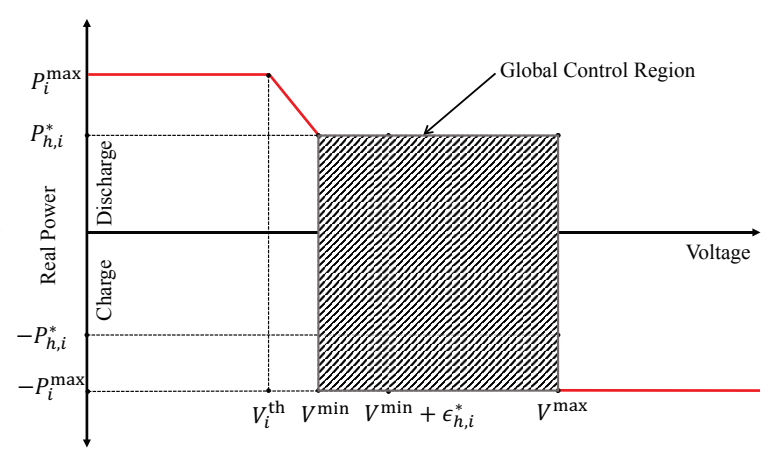

(b)

Figure 2. CVR-based piecewise droop control functions for the inverters of the photovoltaic (PV) systems and energy storage systems (ESSs): (a) Volt-VAR curve; (b) Volt-Watt curve.

Figure $2 \mathrm{a}$, b show the proposed piecewise droop control curves associated with the reactive power outputs of both the PV systems and the ESSs, and to the real power output of the ESSs, respectively. These droop control curves are updated using the optimization solution of the global control scheme, $\left(P_{h, i}^{*}, Q_{h, i}^{*}\right.$, and $\left.\epsilon_{h, i}^{*}\right)$, every hour. In the local control scheme, the power outputs of the PV systems and the ESSs are calculated using the droop curves along with local voltage measurements. If the local voltage measurements are in the acceptable range of voltage (shaded region), the output powers of the PV systems and ESSs remain the same as the solution obtained from the global control scheme. Otherwise, the output powers of the PV systems and ESSs are recalculated based on the droop curves. The proposed Volt-VAR and Volt-Watt droop curves can be expressed, respectively, as follows:

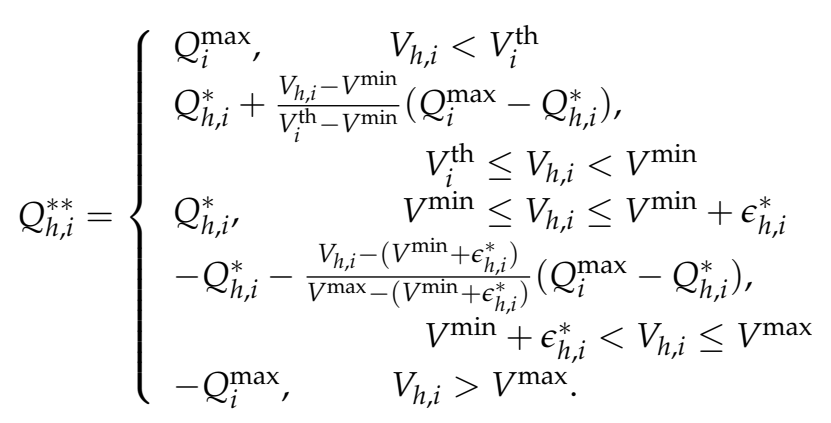

$$
\begin{aligned}
& P_{h, i}^{* *}=\left\{\begin{array}{lc}
P_{i}^{\max }, & V_{h, i}<V_{i}^{\text {th }} \\
P_{h, i}^{*}+\frac{V_{h, i}-V^{\min }}{V_{i}^{\text {th }}-V^{\min }}\left(P_{i}^{\max }-P_{h, i}^{*}\right), \\
V_{i}^{\text {th }} \leq V_{h, i}<V^{\min } \\
P_{h, i^{\prime}}^{*}, & V^{\min } \leq V_{h, i} \leq V^{\max } \\
-P_{i}^{\max }, & V_{h, i}>V^{\max } .
\end{array}\right.
\end{aligned}
$$

As shown in Figure $2 b$, the aforementioned reserved power is associated with only the discharging mode of the ESSs, and thus the Volt-Watt droop curve is more asymmetric than the Volt-VAR droop curve shown in Figure 2a. Even though the voltage deviates from the maximum CVR voltage limit within the acceptable range $\left(V^{\min }+\epsilon_{h, i}^{*} \leq V \leq V^{\max }\right)$, the ESSs never charge real power during the local control process. This design is motivated by our purpose to minimize the real power drawn into the substation and to reduce the number of charging and discharging cycles of the ESSs, considering only the mitigation of upper voltage limit violations (not considering CVR energy savings). However, if Volt-Watt curve is designed with some slope for real power charging process, which is similar to Volt-VAR curve for reactive power absorbing process, more CVR energy savings could be achieved 
at the expense of more frequent charging and discharging cycles of the ESSs outside the range of $V^{\min } \leq V \leq V^{\min }+\epsilon_{h, i}^{*}$.

Lastly, for the local control, we assume that the reactive power from the PV systems and the ESSs can be first used to achieve CVR where the PV systems have a higher priority to regulate voltage than the ESSs. If the amount of injecting and/or absorbing reactive power from the PV systems and the ESSs is not sufficient for voltage regulation, the real power capability of the ESSs is then used.

\section{Simulation Results}

\subsection{Simulation Setup}

In this section, we analyze the performance of the proposed CVR method in the IEEE 33-bus distribution test system [36], which is in Figure 3. In this test system, the base MVA is set to be 100 MVA. To more clearly quantify the impact of VRs and DERs on CVR, the IEEE 33-bus system is modified with the addition of 18 voltage regulating devices, including an OLTC, nine CBs, and four smart inverters for both PV systems and ESSs. The OLTC at the substatioin has a tap position with an integer value $\left(-10 \leq \mathrm{Tap}_{h}^{\mathrm{OLTC}} \leq 10\right)$, and each tap changes $a^{\text {OLTC }}=0.005$ p.u., while maintaining the voltage at the substation within the acceptable voltage range of [0.95 p.u., 1.05 p.u.]. The maximum number of OLTC tap changes and CB switchings during the predicted horizon are set to NTap ${ }^{\text {LLTC,max }}=3$ and $N_{C a p}{ }^{\max }=3$. The CBs are connected to nodes $4,7,8,14,23,24,25,30$, and 32 , and the maximum output of each CB is $30 \mathrm{kVAr}$. The size of the PV systems and the ESSs are $100 \mathrm{kVA}$ and $30 \mathrm{kVA}$, respectively, with a battery capacity of $E_{i}^{\mathrm{ESS}, \max }=100 \mathrm{kWh}$ and are connected to nodes $11,16,27$, and 31. For the ESSs, the maximum charging and discharging power is $30 \mathrm{~kW}$, and the minimum charging and discharging powers is $0 \mathrm{~kW}$.

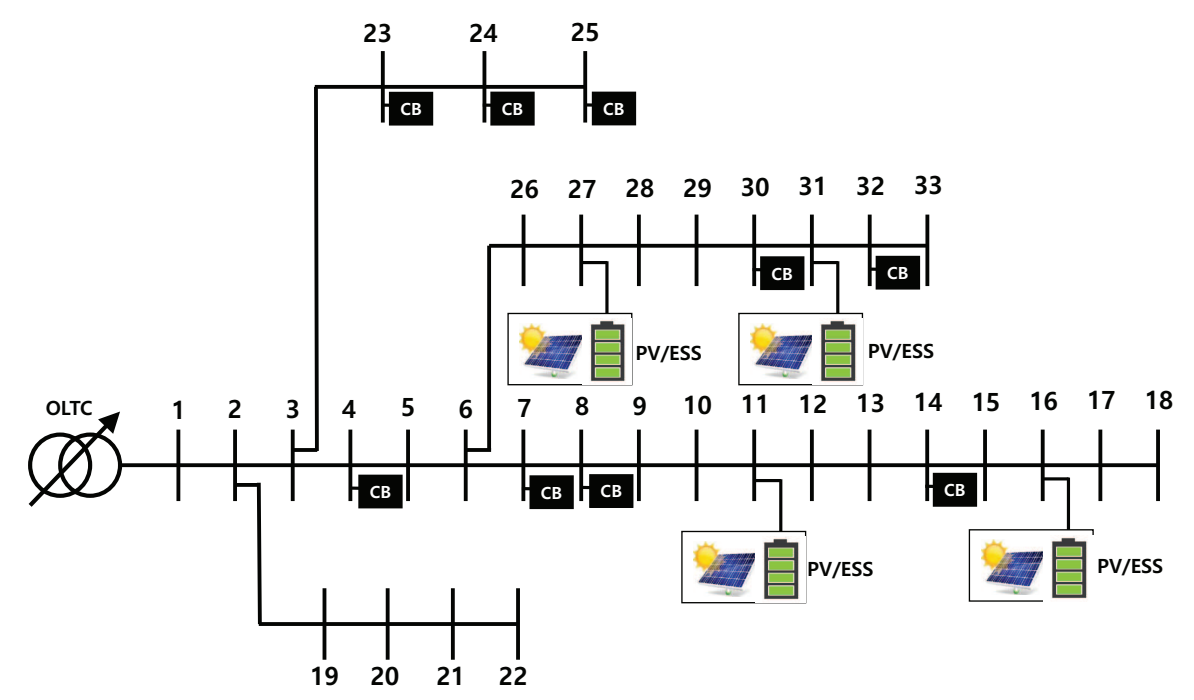

Figure 3. Modified IEEE 33-bus system with on-load tap changer (OLTC), capacitor banks (CBs), PV systems and ESSs.

The initial, minimum, and maximum SOCs are $0.3,0.3$, and 1.0, respectively, and the charging and discharging efficiencies $\eta_{i}^{\text {ch }}$ and $\eta_{i}^{\mathrm{dch}}$ are both $95 \%$. For simplicity, we assume that the predicted load coefficient shown in Figure 4a and the PV generation output $\widehat{P}_{h, i}^{P V}$ shown in Figure $4 \mathrm{~b}$ are identical for all nodes in the test system, and could be obtained accurately. The two exponents of the exponential load model are set to $\alpha=1.5$ and $\beta=3.15$. Furthermore, each consumer is assumed to join the DR program and accepts DR reduction requests.

Initially, we assume that there are no demand reductions, so $X_{h, i}^{\mathrm{DR}}=0$. At the global level, the scheduling period is $1 \mathrm{~h}$ with a predicted horizon $N_{h}=4 \mathrm{~h}$. In the droop control curve at the local level, the voltage threshold, $V_{i}^{\text {th }}$, is set to 0.94 p.u. Numerical testing is performed using the optimization 
toolbox in MATLAB R2015b (MathWorks, Natick, Massachusetts, USA) (Intel Core i5 CPU (Santa Clara, California, USA) clocking at $3.0 \mathrm{GHz}$ and $4 \mathrm{~GB}$ of RAM).

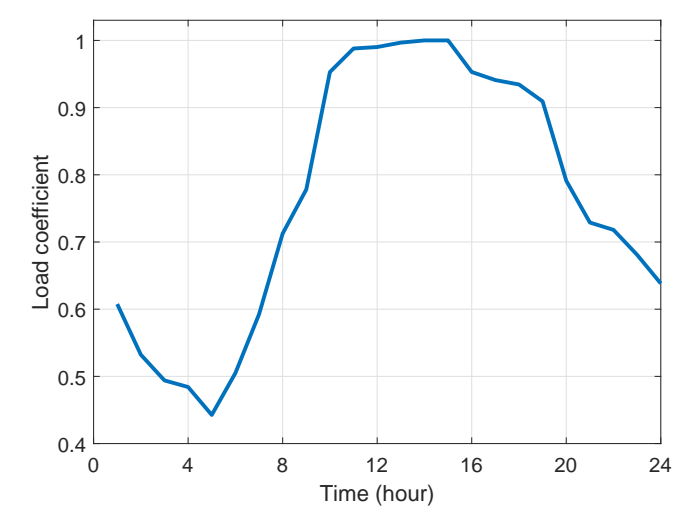

(a)

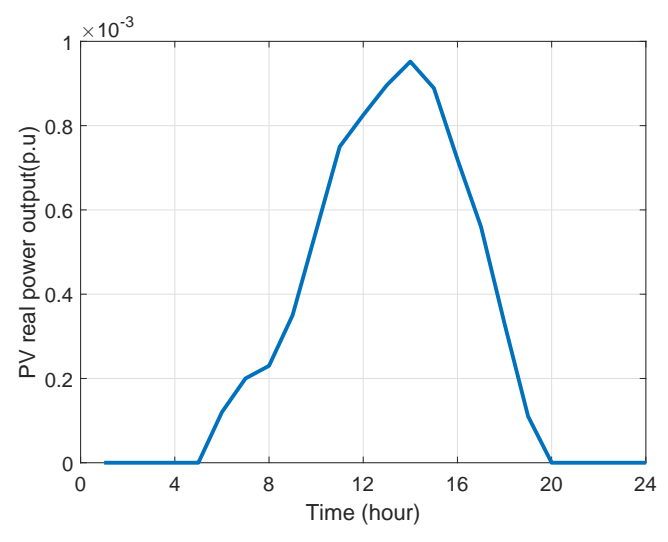

(b)

Figure 4. Profile of the predicted value with a resolution of $1 \mathrm{~h}$ for: (a) load coefficient; (b) PV real power output.

\subsection{Performance Assessment of the Proposed CVR Framework}

In this subsection, we demonstrate the merit of the proposed CVR method in the following three cases:

- Case 1: No CVR with PV systems and ESSs,

- Case 2: CVR without PV systems and ESSs,

- $\quad$ Case 3: CVR with PV systems and ESSs.

In Case 1, a traditional VVO method to maintain voltage within an acceptable range is simulated, for which the objective function Equation (1) is replaced by $\left|V_{h, i}-V^{\text {nom }}\right|$ and the CVR constraint in Equation (2) as well as the SOC regulation constraints in Equations (24), (25), and (26) are deleted. In Case 2, the terms related to the PV systems and the ESSs in Equations (7) and (8) and all the SOC constraints presented in Equations (20), (21), (22), (23), (24), (25), and (26) are deleted. Furthermore, as only OLTC and CBs can regulate feeder voltages in Case 2, the terms related to the reserved power of the PV systems and the ESSs in Equations (27), (28), (29), and (30) are also eliminated. Our proposed algorithm is demonstrated in Case 3. For all three cases, the voltage profiles for all nodes at 2:00 p.m. are compared, as shown in Figure 5. We first verify that the voltage at the end of the feeder (node 18) has the lowest value. In general, the potential voltage drop for CVR is limited by the voltage at node 18. From Figure 5, we can list the three cases in decreasing order of magnitude of voltage: Case $1>$ Case $2>$ Case 3. Compared with Case 1, Cases 2 and 3 show that the proposed CVR-based objective function and constraints explicitly contribute to reduce the voltage profile.

Comparing Cases 2 and 3, we observe that the voltage in Case 3 is lower than in Case 2, and the slope of the voltage drop in the former is more gentle than that in the latter. This is because the injected real and/or reactive power from the PV systems and the ESSs can increase the voltage profile to provide more room for voltage reduction along the feeder, thus allowing the OLTC to more flexibly tap down the voltage at the substation. For example, in Case 2, the voltage at node 18 already hits the lowest limit, so the OLTC could no longer lower the tap position. However, in Case 3, some injecting mode PV systems and ESSs allow the OLTC to reduce the voltage profile along the feeder while the other absorbing mode PV systems and ESSs guarantee that voltage at some node reaches its lowest limit. This phenomenon, which allows for more flexible OLTC operation, is verified as shown in Figure 5, where it is clear that the substation voltage (at node 1) in Case 3 is considerably lower than in the other two cases. 


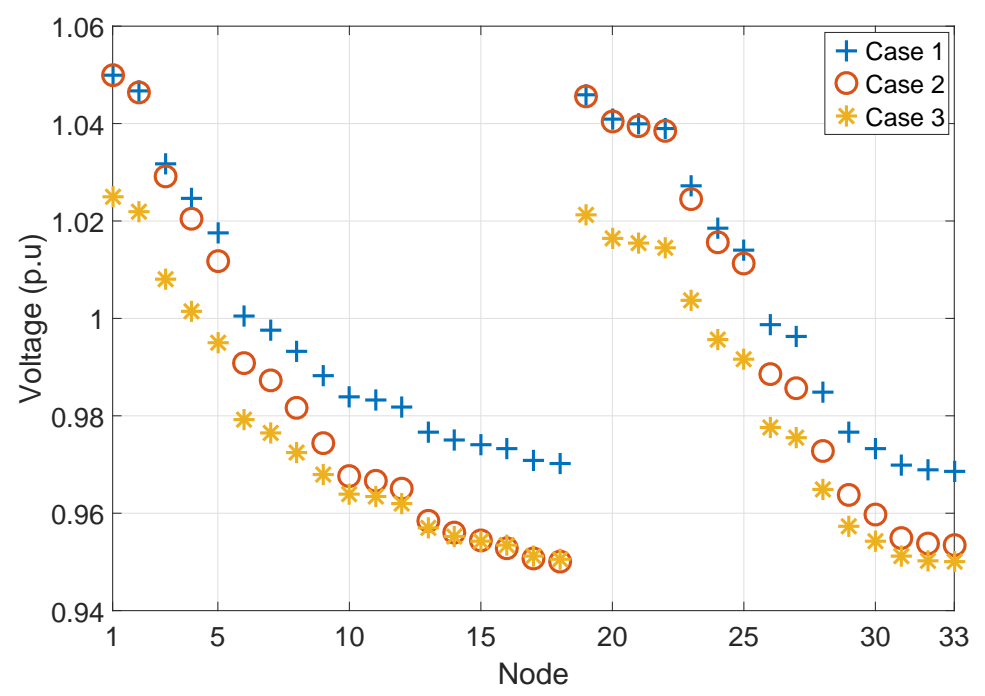

Figure 5. Voltage profiles at 2:00 p.m. in Cases 1, 2, and 3.

Figure 6 shows the injected real and reactive powers at the substation with and without the proposed algorithm. To fairly assess the CVR performance of the proposed approach in the same situation, Case 1 (without CVR) and Case 3 (with CVR) are compared, both of which consider PV systems and ESSs in the distribution test system. As shown in Figure 6, less real and reactive power is consumed in Case 3 at the substation than in Case 1. Compared with Case 1, the scheme used in Case 3 achieves energy savings of real and reactive power of $3.58 \%$ and $3.62 \%$, calculated using the following performance index:

$$
\frac{\sum_{h=1}^{24}\left(X_{h, 1}^{\text {Case } 1}-X_{h, 1}^{\text {Case 3 }}\right)}{\sum_{h=1}^{24} X_{h, 1}^{\text {Case } 1}} \times 100(\%)
$$

where $X$ represents either real power $P$ or reactive power $Q$.

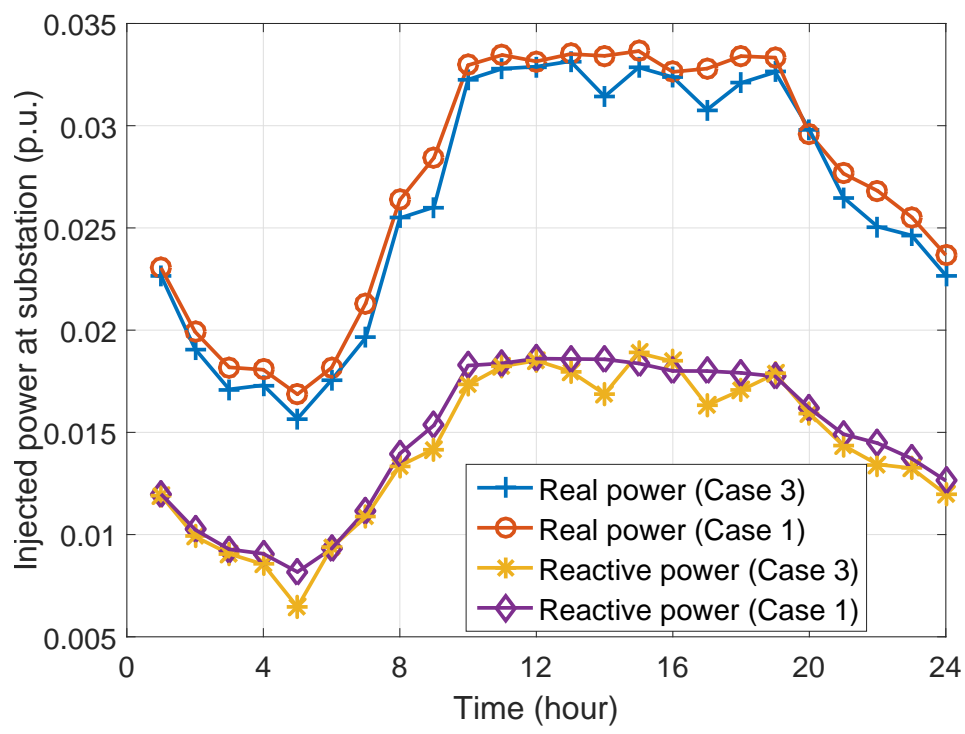

Figure 6. Injected real and reactive power at the substation in Cases 1 and 3. 
Figure 7a-d show the operating schedule of the CBs, the OLTC, the PV systems, and the ESSs for $24 \mathrm{~h}$ in Case 3, respectively. It should be noted that all nine CBs switch on at 5:00 a.m., 2:00 p.m., and 5:00 p.m. as shown in Figure 7a. In these scheduling periods, it is clear from Figure $7 \mathrm{~b}$ that the OLTC selects the lower tap position by changing: (i) from tap position -1 to position -2 at 5:00 a.m.; and (ii) from tap position 6 to position 5 at both 2:00 p.m. and 5:00 p.m. This observation derives from the fact that the CBs can fully support raising the voltage profile using their reactive power, which in turn enables the OLTC to lower the voltage along the feeder to a greater extent. We also verify from Figure $7 \mathrm{c}$ that all four PVs inject their reactive power at the same scheduling periods along with the switch on state of the CBs, increasing their voltage profiles and consequently resulting in an additional voltage reduction from the OLTC. Figure $7 \mathrm{~d}$ compares the scheduled SOC levels of four ESSs during $24 \mathrm{~h}$. We can see from this figure that all the ESSs charge their batteries at 5:00 a.m., which consequently lead to a reduction of the voltage level. This is different from the operation of CBs and PV systems, which aim to increase the voltage profile. Indeed, the loading conditions at 5:00 a.m., shown in Figure $4 \mathrm{a}$ are the lowest during 24 -h period being considered, and thus the corresponding voltage profile is relatively higher than at other times. In this experimental environment, while the OLTC sets its tap position as low as possible, the ESSs further decrease the voltage by absorbing real power. On the other contrary, we see in Figure 7d that most ESSs (except the ESS connected to node 16 at 2:00 p.m.) significantly discharge their batteries at 2:00 p.m. and 5:00 p.m. to assist the OLTC to further reduce voltage at the substation.

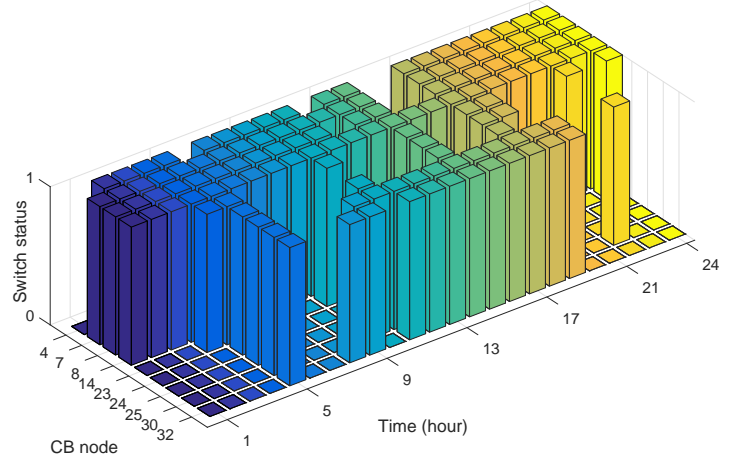

(a)

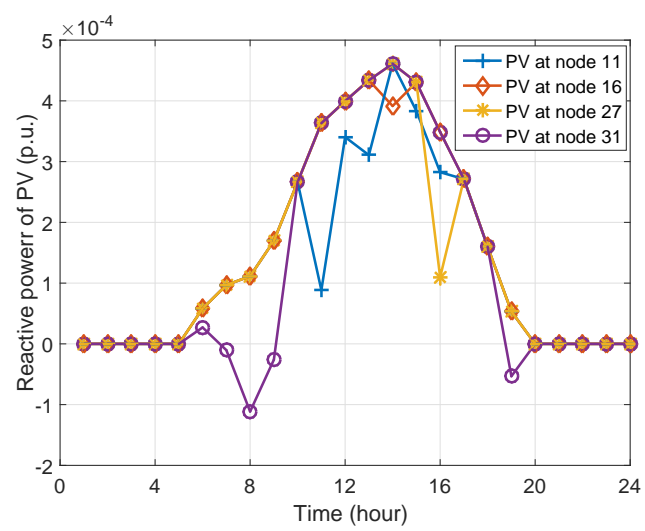

(c)

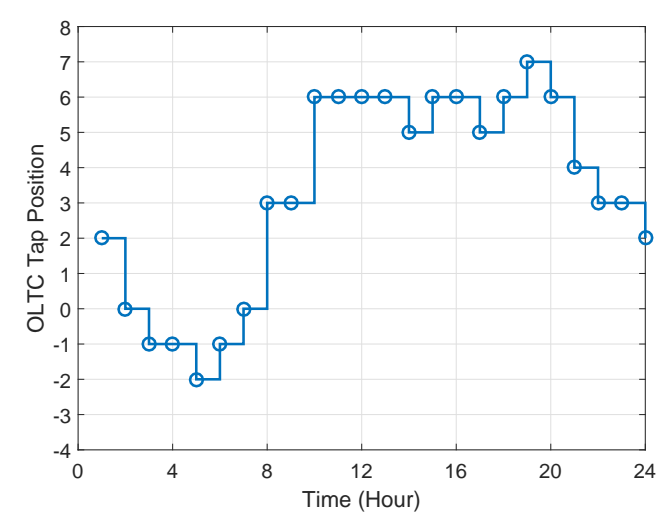

(b)

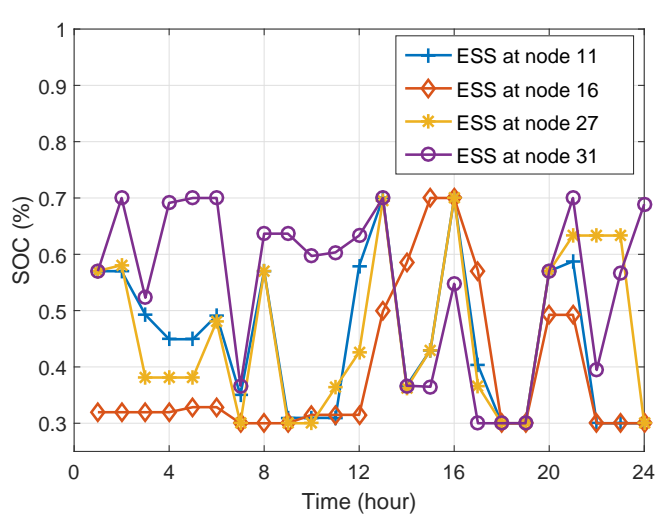

(d)

Figure 7. Operating conditions of voltage regulators and distributed energy resources (DERs) in Case 3: (a) CBs with optimal switch statuses; (b) OLTC with optimal tap positions; (c) PV systems with optimal reactive power output; (d) ESSs with optimal state of charges (SOCs). 
We then evaluate the performance of the local control scheme in Case 3. A 10-min PV real power output profile is used, in which the PV output suddenly drops at 2:00 p.m. as shown in Figure 8. Based on this given profile of the PV output, Figure 9a shows the voltage at nodes 16 and 31 (which have PV systems/ESSs) with and without the local control scheme. We see from this figure that the local control starts to increase the voltage at node 31 after approximately $40 \mathrm{~s}$ when the voltage violation at node 31 is detected.

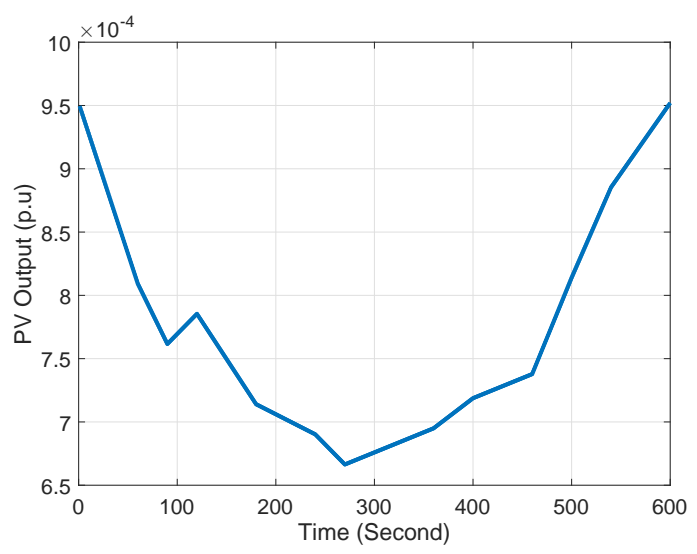

Figure 8. Profile of the predicted PV real power output with a 1-s resolution from 2:00 p.m. to 2:10 p.m.

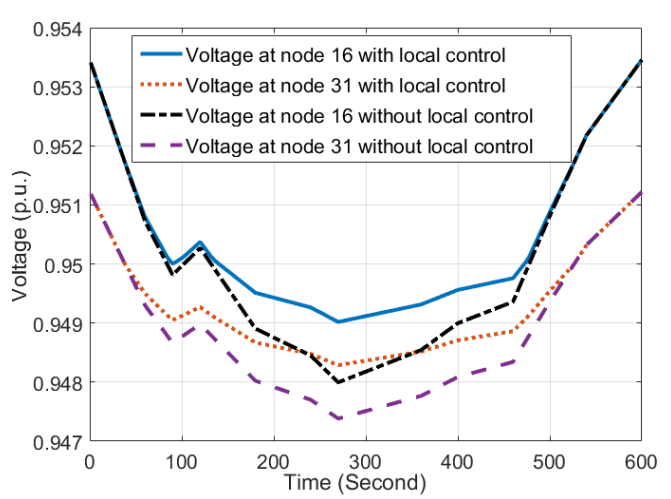

(a)

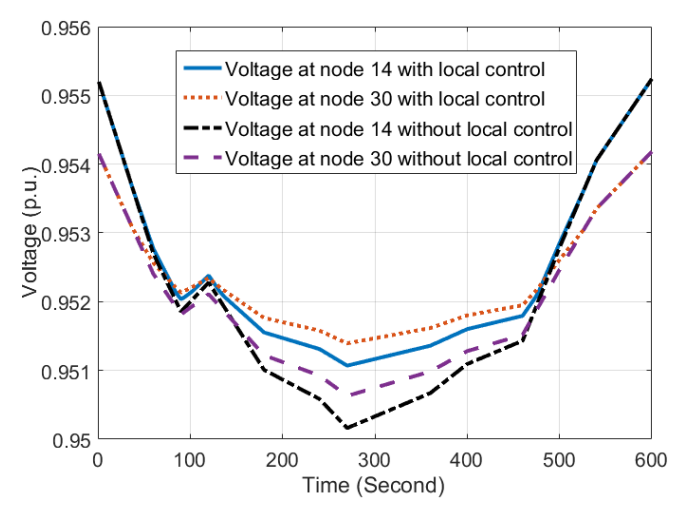

(b)

Figure 9. Comparison of the resulting voltage profiles with and without executing local control scheme for Case 3: (a) nodes 16 and 31 with PV systems/ESSs; (b) nodes 14 and 30 without PV systems/ESSs.

This increase in voltage is obtained via the reactive power support of the DER based on the proposed droop curve. This voltage regulation at node 31 also leads to a slight increase in voltage at node 16 , even though no voltage violation occurs at that node. When the voltage violation is detected at node 16 after approximately $84 \mathrm{~s}$, the local control process initiates at the node. Prior to the execution of the local control process, the lowest voltage values during the PV output fluctuations are 0.9480 p.u. and 0.9474 p.u. for nodes 16 and 31, respectively. Once the local control process is finished, the values of the voltages at nodes 16 and 31 increase to 0.9490 p.u. and 0.9483 p.u., respectively. We can verify from Figure 9a that the proposed local control approach performs well. Finally, Figure $9 \mathrm{~b}$ shows the impact of local control on voltage levels at nodes 14 and 30, which have no PV systems or ESSs. We can also see from this figure that the voltages at these nodes increase slightly when the local control process is executed at the other nodes with PV systems and ESSs.

Figure 10 shows the impact of demand reduction on the voltage profile in the proposed CVR algorithm. For simplicity, we assume that all nodes have identical demand reduction. Figure 10a-C correspond to no reduction, $10 \%$ reduction, and 20\% reduction, respectively, based on the load profile 
shown in Figure 4a. We see from these figures that, as the demand reduction increases, voltage at all nodes during all scheduling periods decreases. This is because demand reduction leads to an increase in the feeder voltage profile so that additional room for voltage reduction in the proposed CVR framework can be achieved. As a result, an optimal coordination between the OLTC, CBs, and DERs in the proposed framework can further lower the voltage profile along the feeder as demand reduction increases. In the traditional VVO framework without CVR, demand reduction may not provide customers with their desired energy savings owing to increases in voltage that trigger following demand reduction. This phenomenon is known as demand response mismatch (DRM) [32]. The adverse effects of DRM on energy saving worsen with increasing customer participation in DR programs. Even under DRM conditions, the proposed CVR approach can achieve energy savings via the reduction of the voltage profiles. As the rate of DR participation increases, the relative energy saving of the proposed method compared with that of no-CVR methods becomes more significant.

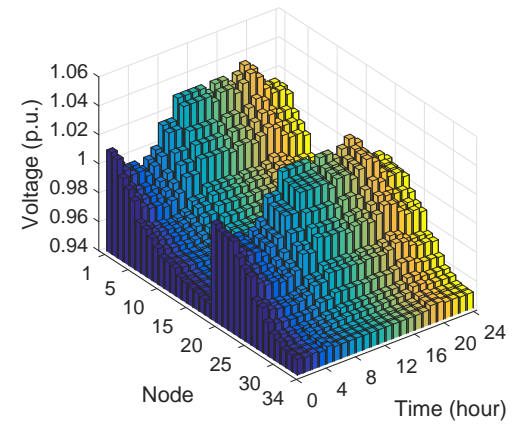

(a)

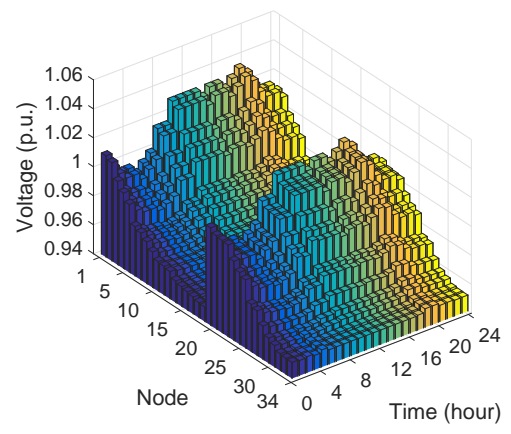

(b)

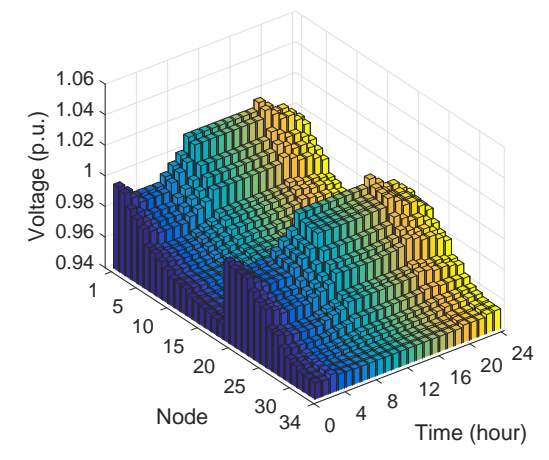

(c)

Figure 10. Voltage profiles with demand reduction for Case 3: (a) $X^{\mathrm{DR}}=0$ ( $0 \%$ reduction); (b) $X^{\mathrm{DR}}=0.1\left(10 \%\right.$ reduction); (c) $X^{\mathrm{DR}}=0.2(20 \%$ reduction) .

Lastly, we emphasize the main contributions of our paper as follows. Much of the recent research on distributed and hierarchical VVO methods have focused on only on maintaining voltage quality (within the acceptable voltage range) without explicitly considering energy savings from CVR. Compared to the existing work, we aim to present the hierarchical CVR approach (a global control and a local control) to lower distribution voltages to allow for consumer energy savings.

First, unlike the existing CVR optimization formulation that minimizes the real power drawn into the substation, our optimization problem in the global control scheme has been formulated to explicitly minimize the value of $\epsilon_{h, i}$ (i.e., positive voltage deviation from its lowest limit) with incorporating its corresponding constraints into the optimization problem. This formulation has been motivated by the principle of CVR. 
Second, we design asymmetric piecewise droop control curves (a Volt-VAR curve for PV systems/ESSs and a Volt-Watt curve for ESSs) that can be used to conduct CVR in the local control scheme. In general, conventional local voltage droop curves are symmetric because they do not consider the operation of CVR. On the other hand, our local droop curves explicitly involve the operation of CVR and become asymmetric by setting the parameters of the optimal solutions from the global control scheme (e.g., $\epsilon_{h, i}$ ) while the global control scheme is executed optimally. To the best of the authors knowledge, this work is the first to develop the CVR algorithm at the local level using the asymmetric local droop curves with the global CVR optimization formulation according to the principle of CVR.

\section{Conclusions}

In this paper, a distributed and hierarchical multi-time scale CVR framework is proposed to achieve energy savings through the reduction of the voltage along the distribution feeder. The proposed framework consists of global and local control schemes. The former reduces the voltage of the entire feeder and achieve energy saving by dispatching hourly real and/or reactive power from CBs, PV systems, and ESSs along with the scheduling of the OLTC tap position in a look-ahead optimization framework. When local voltage violations owing to intermittent PV power output are detected during an hourly dispatch interval, the local control scheme operates based on the proposed droop control functions every second to mitigate voltage violations, achieving further energy savings. Numerical examples simulated in the IEEE 33-bus system confirm the effectiveness of the proposed method in terms of voltage profile reduction along the distribution feeder and energy saving.

In future works, a practical implementation of the proposed CVR approach will be developed and tested in a large-scale realistic unbalanced distribution system. Another interesting direction for future research is to investigate the optimal location of the DERs and CBs to achieve the highest energy savings via CVR from the perspective of power system planning.

Author Contributions: D.M. proposed the distributed CVR model and simulated the proposed model. D.-H.C. coordinated the proposed approach in this paper.

Funding: This work was supported in part by the National Research Foundation of Korea (NRF) grant funded by the Korea government (MSIP) (No. 2018R1C1B6000965), in part by the Korea Electric Power Corporation (No. R17XA05-75), and in part by the Human Resources Development (No.20184030202070) of the Korea Institute of Energy Technology Evaluation and Planning (KETEP) grant funded by the Korea government Ministry of Trade, Industry and Energy.

Conflicts of Interest: The authors declare no conflict of interest.

\section{Abbreviations}

The following abbreviations are used in this manuscript:

$\begin{array}{ll}\text { CVR } & \text { Conservation Voltage Reduction } \\ \text { VVO } & \text { Volt/VAR Optimization } \\ \text { PV } & \text { Photovoltaic } \\ \text { ESS } & \text { Energy Storage System } \\ \text { SOC } & \text { State of Charge } \\ \text { DER } & \text { Distributed Energy Resource } \\ \text { OLTC } & \text { On-Load Tap Changer } \\ \text { SVR } & \text { Step-Type Voltage Regulator } \\ \text { CB } & \text { Capacitor Bank } \\ \text { DR } & \text { Demand Response } \\ \text { DRM } & \text { Demand Response Mismatch } \\ \text { MILP } & \text { Mixed-Integer Linear Programming }\end{array}$




\section{Nomenclature}

The main notations for the proposed hierarchical CVR method are summarized. The other undefined symbols are explained in the text:

$\epsilon_{h, i} \quad$ Voltage magnitude deviation from the lowest voltage limit for node $i$ at period $h$

$\zeta_{h, i} \quad$ Deviation of SOC from its middle level for node $i$ at period $h$

$r_{i} \quad$ Resistance of line $i$

$x_{i} \quad$ Reactance of line $i$

$\alpha \quad$ Exponent coefficient of real power load for exponent load model

$\beta \quad$ Exponent coefficient of reactive power load for exponent load model

$P$ line $\quad$ Real power flow from node $i$ to $i+1$ at period $h$

$Q_{h, i}^{\text {line }} \quad$ Reactive power flow from node $i$ to $i+1$

$P_{h, i}^{\text {node }} \quad$ Net real power consumption for node $i$ at period $h$

$Q_{h, i}^{\text {node }} \quad$ Net reactive power consumption for node $i$ at period $h$

$P(Q)_{h, i}^{\text {lat }} \quad$ Real (Reactive) power flow through the lateral branch from node $i$ at period $h$

$V_{h, i} \quad$ Voltage for node $i$ at period $h$

$V_{h, i}^{k}$

$V_{h, i}^{k, \max }$

$\widehat{P} \widehat{P}_{h, i}$

$Q_{h, i}^{P V}$

$P_{h, i}^{\mathrm{ESS}, \mathrm{ch}(\mathrm{dch})}$

$Q_{h i}^{\mathrm{ESS}}$

$P_{i}^{\mathrm{ESS}, \mathrm{i}, \mathrm{ch}, \max }$

$P_{i}^{\mathrm{ESS}, \mathrm{ch}, \min }$

Voltage of piecewise linear function in the $k$ th piecewise interval for node $i$ at period $h$

Maximum voltage of piecewise linear function in the $k$ th piecewise interval for node $i$ at period $h$

Predicted PV real power output for node $i$ at period $h$

PV reactive power output for node $i$ at period $h$

Charging (Discharging) real power of ESS for node $i$ at period $h$

Discharging reactive power of ESS for node $i$ at period $h$

$P_{i}^{\mathrm{ESS}, \mathrm{dch}, \max }$

$P_{i}^{\text {ESS,dch,min }}$

$E_{i}^{\mathrm{ESS}, \max }$

$\mathrm{SOC}_{h, i}$

$S O C_{h, i}^{\max (\min )}$

$\mathrm{SOC}_{i}^{\text {mid }}$

$\eta_{i}^{\mathrm{ch}(\mathrm{dch})}$

$Q^{\text {res }}$

Pres

$V^{\max (\min )}$

$Q_{i}^{\text {CAP,nom }}$

$N_{\text {Tap }}$ OLTC

Maximum charging real power of ESS for node $i$

Minimum charging real power of ESS for node $i$

Maximum discharging real power of ESS for node $i$

Minimum discharging real power of ESS for node $i$

Maximum energy capacity of ESS for node $i$

State of the charge of ESS for node $i$ at period $h$

Maximum (Minimum) charge state of ESS for node $i$ at period $h$

Mid-level of state of the charge of ESS for node $i$

Charging (Discharging) efficiency of ESS for node $i$

Reserved reactive power

Reserved real power

Maximum (Minimum) allowed voltage

$\mathrm{NCap}_{h, i}$

$Q_{h, i}^{\mathrm{CAP}}$

Pload

$P_{h, i}^{\text {load }}$

$Q_{h, i}^{\text {load }}$

Pload,nom

$Q_{h, i}^{\text {load,nom }}$

Nominal reactive power output of CB for node $i$

Number of OLTC tap operations at period $h$

$X_{h, i}^{\mathrm{DR}}$

$S^{P(Q)}$

$S_{h, i, k}$

$\Delta V_{h, i}^{k}$

Tap OLTC

$a^{\text {OLTC }}$

$b_{h, i}^{\text {Cap }}$

NTap $\max$ OLC

Number of switch operations for node $i$ at period $h$

Reactive power output of CB for node $i$ at period $h$

Real load consumption for node $i$ at period $h$

Reactive load consumption for node $i$ at period $h$

Real load consumption at nominal voltage for node $i$ at period $h$

Reactive load consumption at nominal voltage for node $i$ at period $h$

Load reduction ratio in $[0,1]$ by demand response for node $i$ at period $h$

Slope of the $k$ th piecewise linear block for real(reactive) power for node $i$ at period $h$

Increment of $V_{h, i}$ in the $k$ th piecewise interval

Tap position of OLTC at period $h$

Step size of change in OLTC tap position

Binary switch status of the capacitor for node $i$ at period $h$; " 1 " for ON and "0" OFF

Maximum switching operations of OLTC during the prediction horizon $N_{h}$

NCap $\max$

$b_{h, i}^{\mathrm{ESS}}$

$N_{b}$

Maximum switching operations of capacitor during the prediction horizon $N_{h}$

Binary charging state of ESS for node $i$ at period $h . ;$ " 1 " for charging and " 0 " otherwise

Number of nodes

$N_{h} \quad$ Number of prediction horizon 


\section{References}

1. Roytelman, I.; Medina, J. Volt/var control and conservation voltage reduction as a function of advanced DMS. In Proceedings of the 2016 IEEE Power \& Energy Society Innovative Smart Grid Technologies Conference (ISGT), Minneapolis, MN, USA, 1-4 September 2016.

2. Basso, T.S. IEEE 1547 and 2030 Standards for Distributed Energy Resources Interconnection and Interoperability with the Electricity Grid. Tech. Rep., NREL/TP-5D00-63157, December 2014. Available online: https: / /www.nrel.gov/docs/fy15osti/63157.pdf (accessed on 20 May 2017).

3. Rahimi, K.; Tbaileh, A.; Broadwater, R.; Woyak, J.; Dilek, M. Voltage regulation performance of smart inverters: Power factor versus volt-var control. In Proceedings of the 2017 North American Power Symposium (NAPS), Morgantown, WV, USA, 1-6 September 2017.

4. Weckx, S.; Gonzalez, C.; Driesenh, J. Combined central and local active and reactive power control of PV inverters. IEEE Trans. Sustain. Energy 2014, 5, 776-784. [CrossRef]

5. Preiss, R.F.; Warnock, V.J. Impact of voltage reduction on energy and demand. IEEE Trans. Power Appl. Syst. 1978, PAS-97, 1665-1671. [CrossRef]

6. Scalley, B.R.; Kasten, D.G. The effects of distribution voltage reduction on power and energy consumption. IEEE Trans. Educ. 1981, E-24, 210-216. [CrossRef]

7. Wang, Z.; Wang, J. Review on implementation and assessment of conservation voltage reduction. IEEE Trans. Smart Grid 2014, 29, 1306-1315. [CrossRef]

8. Sen, P.K.; Lee, K.H. Conservation voltage reduction technique: An application guideline for smarter grid. IEEE Trans. Ind. Appl. 2016, 52, 2122-2128. [CrossRef]

9. Ahmadi, H.; Martí, J.R.; Dommel, H.W. A framework for volt-var optimization in distribution systems. IEEE Trans. Smart Grid 2015, 6, 1473-1483. [CrossRef]

10. Wang, Z.; Wang, J.; Chen, B.; Begovic, M.M. MPC-based voltage/var optimization for distribution circuits with distributed generators and exponential load models. IEEE Trans. Smart Grid 2014, 5, 2412-2420. [CrossRef]

11. Wang, Z.; Chen, B.; Wang, J.; Begovic, M.M. Stochastic DG placement for conservation voltage reduction based on multiple replications procedure. IEEE Trans. Power Deliv. 2015, 30, 1039-1047. [CrossRef]

12. Zhang, Y.; Ren, S.; Dong, Z.Y.; Xu, Y.; Meng, K.; Zheng, Y. Optimal placement of battery energy storage in distribution networks considering conservation voltage reduction and stochastic load composition. IET Gener. Transm. Distrib. 2017, 11, 3862-3870. [CrossRef]

13. Gheydi, M.; Nouri, A.; Ghadimi, N. Planning in microgrids with conservation of voltage reduction. IEEE Syst. J. 2018, 12, 2782-2790. [CrossRef]

14. Wang, J.; Raza, A.; Hong, T.; Sullberg, A.C.; de León, F.; Huang, Q. Analysis of energy savings of CVR including refrigeration loads in distribution systems. IEEE Trans. Power Deliv. 2018, 33, 158-168. [CrossRef]

15. McKenna, K.; Keane, A. Open and closed-loop residential load models for assessment of conservation voltage reduction. IEEE Trans. Power Syst. 2017, 33, 2995-3005. [CrossRef]

16. Zhao, J.; Wang, Z.; Wang, J. Robust time-varying load modeling for conservation voltage reduction assessment. IEEE Trans. Smart Grid 2018, 9, 3304-3312. [CrossRef]

17. Robbins, B.A.; Domínguez-García, A.D. Optimal reactive power dispatch for voltage regulation in unbalanced distribution systems. IEEE Trans. Power Syst. 2016, 31, 2903-2913. [CrossRef]

18. Daratha, N.; Das, B.; Sharmac, J. Coordination between OLTC and SVC for voltage regulation in unbalanced distribution system distributed generation. IEEE Trans. Smart Grid 2014, 29, 289-299. [CrossRef]

19. Wang, Z.; Chen, H.; Wang, J.; Begovic, M.M. Inverter-less hybrid voltage/var control for distribution circuits with photovoltaic generators. IEEE Trans. Smart Grid 2014, 5, 2718-2728. [CrossRef]

20. Abessi, A.; Vahidinasab, V.; Ghazizadeh, M.S. Centralized support distributed voltage control by using end-users as reactive power support. IEEE Trans. Smart Grid 2016, 7, 178-188. [CrossRef]

21. Wu, H.; Huang, C.; Ding, M. Cooperative strategy for distributed voltage control in active distribution feeders. In Proceedings of the 2017 IEEE 12th International Conference on Power Electronics and Drive Systems (PEDS), Honolulu, HI, USA, 12-15 December 2017; pp. 125-130.

22. Safavizadeh, A.; Yousefi, G.R.; Ghazizadeh, M.S. Voltage variation mitigation using reactive power management of distributed energy resources in a smart distribution system. IEEE Trans. Smart Grid 2018. [CrossRef] 
23. Calderaro, V.; Conio, G.; Galdi, V.; Massa, G.; Piccolo, A. Optimal decentralized voltage control for distribution systems with inverter-based distributed generators. IEEE Trans. Power Syst. 2014, 29, 230-241. [CrossRef]

24. Wang, L.; Yan, R.; Saha, T.K. Voltage management for large scale PV integration into weak distribution systems. IEEE Trans. Smart Grid 2018, 9, 4128-4139. [CrossRef]

25. Liu, X.; Aichhorn, A.; Liu, L.; Lih, H. Coordinated control of distributed energy storage system with tap changer transformers for voltage rise mitigation under high photovoltaic penetration. IEEE Trans. Smart Grid 2012, 3, 897-906. [CrossRef]

26. Kabir, M.N.; Mishra, Y.; Ledwich, G.; Dong, Z.Y.; Wong, K.P. Coordinated control of grid-connected photovoltaic reactive power and battery energy storage systems to improve the voltage profile of a residential distribution feeder. IEEE Trans. Ind. Inf. 2014, 10, 967-977. [CrossRef]

27. Wang, L.; Bai, F.; Yan, R.; Saha, T.K. Real-time coordinated voltage control of pv inverters and energy storage for weak networks with high PV penetration. IEEE Trans. Power Syst. 2018, 33, 3383-3395. 2018.2789897. [CrossRef]

28. Zeraati, M.; Golshan, M.E.H.; Guerrero, J.M. Distributed control of battery energy storage systems for voltage regulation in distribution networks with high PV penetration. IEEE Trans. Smart Grid 2018, 9, 3582-3593. [CrossRef]

29. Zhang, C.; Xu, Y.; Dong, Z.; Ravishankar, J. Three-stage robust inverter-based voltage/var control for distribution networks with high-level PV. IEEE Trans. Smart Grid 2018. [CrossRef]

30. Olivier, F.; Aristidou, P.; Ernst, D.; Cutsem, T.V. Active management of low-voltage networks for mitigating overvoltages due to photovoltaic units. IEEE Trans. Smart Grid 2016, 7, 926-936. [CrossRef]

31. Chou, H.-M.; Xie, L.; Enjeti, P.; Kumar, P.R. Power electronics intelligence at the network edge (pine). In Proceedings of the 2017 IEEE Energy Conversion Congress and Exposition (ECCE), Cincinnati, OH, USA, 19-22 November 2017; pp. 5214-5221.

32. Rahiman, F.A.; Zeineldin, H.H.; Khadkikar, V.; Kennedy, S.W.; Pandi, V.R. Demand response mismatch (DRM): Concept, impact analysis, and solution. IEEE Trans. Smart Grid 2014, 5, 1734-1743. [CrossRef]

33. Bajool, R.; Shafie-khah, M.; Gazafroudi, A.S.; Catalao, J.P.S. Mitigation of active and reactive demand response mismatches through reactive power control considering static load modeling in distribution grids. In Proceedings of the 2017 IEEE Conference on Control Technology and Applications (CCTA), Kohala Coast, HI, USA, 27-30 August 2017; pp. 1637-1642. [CrossRef]

34. Acharya, S.; El Moursi, M.S.; Al-Hinai, A.; Al-Sumaiti, A.S.; Zeineldin, H. A control strategy for voltage unbalance mitigation in an islanded microgrid considering demand side management capability. IEEE Trans. Smart Grid 2018. [CrossRef]

35. Solanki, J.; Venkatesan, N.; Solanki, S.K. Coordination of demand response and volt/var control algorithm using multi agent system. In Proceedings of the 2012 IEEE/PES Transmission \& Distribution Conference and Exposition (T\&D), Orlando, FL, USA, 1-4 May 2012; doi:10.1109/TDC.2012.6281544. [CrossRef]

36. Baran, M.E.; Wu, F.F. Network reconfiguration in distribution systems for loss reduction and load balancing. IEEE Trans. Power Deliv. 1989, 4, 1401-1407. [CrossRef]

(C) 2018 by the authors. Licensee MDPI, Basel, Switzerland. This article is an open access article distributed under the terms and conditions of the Creative Commons Attribution (CC BY) license (http://creativecommons.org/licenses/by/4.0/). 